\title{
SENSITIVITY OF BLIND ADAPTIVE LMMSE SINGLE-USER RECEIVERS TO PATH DELAY AND PHASE ESTIMATION ERRORS IN MULTIPATH FADING CHANNEL
}

\author{
L.Mucchi $^{(1 / 2)}$, M. Latva-aho ${ }^{(1)}$, E. Del Re ${ }^{(2)}$, R. Fantacci $^{(2)}$. \\ 1) Centre for Wireless Communications - University of Oulu (Finland) \\ Tutkijantie 2 E, FIN-90014 Oulu (Finland) \\ 2) Laboratorio di Elaborazione Numerica dei Segnali e Telematica - University of Firenze (Italy) \\ Via di S. Marta, 3 1-50143 Firenze (Italy) \\ Phone: +358-055-4796271 \\ Fax: +39-055-4796485 \\ E-mail to: lorenzo@ieee.org
}

\begin{abstract}
Carrier frequency, carrier phase, multipath profile, delay values as well as fading characteristics are important in a coherent detection. Several DS-CDMA receivers have been investigated, but mainly with the assumption of perfect estimation of those parameters. This paper deals with the impact of channel phase and path delay estimation errors to the performance of Pre and Post-combining blind adaptive Linear Minimum Mean Square Error (LMMSE) receivers. Comparisons are made also with the conventional RAKE receiver. The results show that channel phase estimation errors degrade almost equally the performance of blind adaptive LMMSE and RAKE receivers. Delay estimation errors by over $20 \%$ of chip time cause the loss of advantages of LMMSE detection.
\end{abstract}

\section{INTRODUCTION}

In case of multipath propagation a RAKE receiver is commonly used. It consists of a bank of parallel filters matched to the signature waveform properly delayed according to the propagation delay of a specific path. This detection scheme follows a single-user strategy according to which each path is detected separately regardless of other users or other paths. Despite its low complexity, all signature waveforms should have low cross-correlations and power levels of the received users signals cannot be too much dissimilar (near-far effect $)^{\prime}$, in order to mitigate the Multiple Access Interference (MAI). Unfortunately, in frequencyselective time-varying channels, e.g., mobile radio

The near-far effect can be avoided by the implementation of a strict power control with consequent complexity overhead. channel, these conditions are not fulfilled. Thus, the conventional detector is said to be interference limited. Advanced detection techniques $[5,8]$ have been proposed to cope with near-far effect and nonorthogonal spreading code scenario. Most of these receivers are based on coherent detection, where the user path delays and signal amplitude and phase must be accurately recovered. In order to despread the signal, the locally generated signature waveform has to be time aligned within a fraction of one chip period with the received replica of the desired signal. Then, channel compensation of every signal replica should be performed in order to take advantage from multipath diversity (Maximal Ratio Combining).

In forward link transmission much attention has been paid to blind adaptive detection approaches [5] which do not require knowledge of interfering user signature waveforms, timing and complex amplitudes. These techniques are based on the minimization of the mean square error between detector output and data transmitted [6]. Linear MMSE receivers are less complex, easier to adapt and require less information about the channel parameters and the interferers than many other multiuser receivers. Moreover it is able to alleviate the stringent requirements on power control in DS-CDMA. In the absence of dispersive channels, an MMSE receiver can be obtained by minimizing the output energy subject to a constraint [1], so avoiding the use of training sequences. Unfortunately, this approach is very sensitive to possible signature mismatch caused by dispersive channels, multipath effects or timing errors. Two different schemes are here considered in order to maintain the advantages of blind MMSE approach in multipath channels. Blind adaptive MMSE techniques have been heavily investigated in recent years, but often with the assumption of perfect knowledge of channel parameters and path delay 
recovering. So, it is important to find out if these receivers are able to maintain near-far resistance and MAI rejection capabilities in presence of these errors. In this paper, we investigate, via computer simulations, the robustness of Pre and Post-combining blind adaptive LMMSE single-user detectors (PreBA-SUD, PostBA-SUD) and conventional RAKE detector (CRD) respect to channel parameter and synchronization errors in frequency-selective Rayleigh fading channel. Only forward link is considered.

The organization of this paper is the following: in section II is describe the system model; section III gives a description of the proposed LMMSE receivers and in section IV the numerical results are discussed.

\section{SYSTEM MODEL}

The equivalent baseband received signal $r(t)$ for a CDMA downlink system with $K$ users can be represented as:

$r(t)=\sum_{l=1}^{L} \sum_{n=0}^{N_{b}} \sum_{k=1}^{K} c_{l}{ }^{(n)} A_{k} b_{k}{ }^{(n)} s_{k}\left(t-n T-\tau_{l}\right)+n(t)$

where

- $L$ is the number of resolvable paths,

- $N_{b}$ is the number of transmitted symbols,

- $n(t)$ is the white Gaussian noise with double side power spectral density $N_{t} / 2$,

- $T$ is the symbol interval,

- $A_{k}$ is the amplitude of the $k$-th user,

- $\quad b_{k}^{(n)}$ is the $n$-th bit of $k$-th transmission: they are i.i.d. random values in $\{-1,1\}$ with equal probability 0.5

- $c_{l}^{(n)}$ and $\tau_{1}^{(n)}$ are the channel coefficient and delay characterizing the $l$-th path.

Channel coefficients $c_{l}^{(n)}$ are defined as:

$c_{i}^{(n)}=|c|_{1}^{(n)} \cdot e^{j \phi t^{(n)}}$

where $|c|_{t}^{(n)}$ are i.i.d. Rayleigh distributed random values and $\phi_{l}^{(n)}$ are i.i.d. uniform random values in $[0$, $2 \pi)$.

User $k$ signature waveform $s_{k}(t)$ is defined, upon one symbol interval, as in the following

$s_{k}(t)=\sum_{m=1}^{G} p_{k}(m) u\left(t-m T_{c}\right)$

where $\mathrm{G}=T / T_{t^{\prime}}$ is the processing gain, $T_{c}$ is the chip interval, $p_{k}(m) \in\{ \pm 1\}$ is the $m$-th chip of the $k$-th spreading code and $u(t)$ is the root raised cosine pulse shaping filter impulse response [3], with a roll-off factor of 0.22 . The length of the filter has been truncated to five chip intervals.

Since the bandwidth of practical communications systems is limited, the pulse-shaping filter is employed to reduce ISI. Assuming user of interest to be the first $(k=1)$, the associated bit sequence $\left\{b_{l}{ }^{(n)}\right\}$ has to be demodulated only by means of the signature waveform and timing. No assumption is made about interfering users.

\section{PRE and POST COMBINIG BLIND ADAPTIVE SINGLE-USER LMMSE RECEIVERS}

The well-known Blind Adaptive MultiUser Detector (BA-MUD) of [1] achieves very good performance in a time-invariant environment, but undergoes growing impairments in time-varying multipath fading environments. To overcome this drawback, two different approaches are here proposed depending on the order in which interference suppression and multipath combining are performed. A possible approach is to insert a blind adaptive LMMSE receiver in each finger of a RAKE structure (Fig. 1) in order to counteract MAI effect in each desired signal significant path [9]. We called this detector PreBA-SUD because interference suppression is performed before multipath combining. On the contrary, in PostBA-SUD here proposed (Fig. 2) interference suppression and multipath combining are done simultaneously and only a single filter is needed yielding remarkable complexity reduction.

The received signal is time-discretized at the rate $T_{s}^{-1}=\frac{S G}{T}$ where $S$ is the number of samples per chip.

The received vector, over a single symbol interval, can be expressed as:

$$
\begin{aligned}
& \mathbf{r}^{(n)}=\mathbf{S}^{(n)} \mathbf{C}^{(n)} \mathbf{A} \mathbf{b}^{(n)}+\mathbf{n}^{(n)} \\
& \text { where } \\
& \mathbf{S}^{(n)}=\left[\mathbf{s}_{1,1}^{(n)}, \ldots, \mathbf{s}_{1, L}^{(n)}, \ldots, \mathbf{S}_{K, L}^{(n)}\right] \\
& \mathbf{s}_{k, l}^{(n)}=\left[\begin{array}{c}
\mathbf{0}_{\left[(n-1) S G+\tau_{l}\right] \times 1} \\
\mathbf{s}_{k} \\
\mathbf{0}_{\left[n S G+\left(\tau_{l},-\tau_{l}\right)\right] \times 1}
\end{array}\right]
\end{aligned}
$$

and $\mathbf{s}_{k}=\left[s_{k}\left(T_{s}\right), \ldots, s_{k}\left(S G T_{s}\right)\right]^{T}$. We have supposed, without loss of generality, $0=\tau_{1}<\tau_{2}<\ldots<\tau_{L}<T$.

Channel matrix is:

$\mathrm{C}^{(n)}=\operatorname{diag}\left\{\mathbf{c}^{(n)}, \ldots, \mathbf{c}^{(n)}\right\}$

where $\mathbf{c}^{(n)}=\left(c_{1}^{(n)}, \ldots, c_{L}^{(n)}\right)^{T}=\left(\alpha_{1}^{(n)} e^{\phi_{1}^{(n)}}, \ldots, \alpha_{L}^{(n)} e^{\phi_{i}^{(n)}}\right)^{T}$ is the vector of channel coefficients, $\mathbf{A}=\operatorname{diag}\left\{A_{1}, \ldots, A_{K}\right\}$ is the diagonal matrix of transmitted amplitudes,

$\mathbf{b}^{(n)}=\left(b_{1}^{(n)}, \ldots, b_{K}^{(n)}\right)^{T}$ is the vector of transmitted data and $\mathbf{n}^{(\prime)}$ is the AWG noise vector. 
The received vector is time-aligned to each estimated path delay and then passed through a chip-matched filter ${ }^{2}$ (CMF). The output of each CMF is downsampled at chip rate, multiplied by the complex conjugate of the estimated channel parameter and summed up (maximal ratio combining) (Fig. 2).

The decision variable of the PostBA-SUD, during the n-th symbol interval, is:

$$
\begin{aligned}
& \mathbf{y}_{p(n) s t}^{(m)}=\mathbf{W}_{1}^{(n)}(0) \mathbf{C}^{(n)} \hat{\mathbf{C}}^{H^{(n)}} \mathbf{A} \mathbf{b}^{(n)}+ \\
& +\mathbf{W}_{1}^{(n)}(+1) \mathbf{C}^{(n-1)} \hat{\mathbf{C}}^{\prime^{(n)}} \mathbf{A} \mathbf{b}^{(n-1)}+ \\
& +\mathbf{W}_{1}^{(n)}(-1) \mathbf{C}^{(n+1)} \hat{\mathbf{C}}^{\left({ }^{(n)}\right.} \mathbf{A} \mathbf{b}^{(n+1)}+\mathbf{z}^{(n)} \\
& \text { where } \\
& \mathbf{W}_{1}^{(n)}(i)=\left\{\mathbf{W}_{1.1}^{(n)}(i), \ldots \ldots, \mathbf{W}_{K . !}^{(n)}(i)\right\}
\end{aligned}
$$

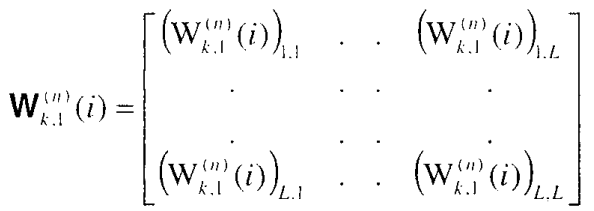

$$
\begin{aligned}
& \left(W_{k .1}^{(n)}(i)\right)_{1, l^{\prime}}=\int_{-\infty}^{+\infty} s_{k}^{(n-i)}\left(t+i T-\tau_{l}+\hat{\tau}_{l^{\prime}}\right)\left[s_{1}(t)+x_{i}^{(n)}(t)\right] d t \\
& \hat{\mathbf{C}}^{(n)}=\operatorname{diag}\left\{\hat{\mathbf{c}}^{(n)}, \ldots, \hat{\mathbf{c}}^{(n)}\right\}
\end{aligned}
$$

$\hat{\mathbf{c}}^{(n)}=\left(\hat{c}_{1}^{(n)}, \ldots, \hat{c}_{L}^{(n)}\right)^{T}$ are the channel estimated coefficients and $\mathbf{z}^{(n)}$ is the output noise vector.

It is important to notice that $\hat{\tau}_{l^{\prime}}$ and $\hat{c}_{l}^{(n)}$ are the estimated path delay and channel coefficient, respectively. In order to evaluate the sensitivity to channel estimation errors, the detectors are assumed working with a delay

$\hat{\tau}_{r^{\prime}}=\tau_{r^{\prime}}+\Delta \tau_{r^{\prime}}$

and a phase

$$
\hat{\phi}_{l}^{(n)}=\phi_{l}^{(n)}+\Delta \phi_{l}^{(\prime)}
$$

where $\Delta \tau_{f^{\prime}}$ is the synchronization error relative to one chip period and $\Delta \phi_{l}^{(n)}$ is the absolute phase estimation error. We have applied a constant synchronization error to all users. In practical system all users will not experience the same synchronization error and this scenario can be therefore considered as a worst case. In the same manner we assume that all users experience the same amount of phase estimation error.

The receiver vector can be decomposed into fixed and adaptive components:

$$
\mathbf{w}_{1}^{(n)}=\mathbf{s}_{1}+\mathbf{x}_{1}^{(n)}
$$

\footnotetext{
'The signal at the output of CMF could be viewed as vector whose
} dimension is equal to the number of samples per symbol [1]
Many algorithms can be used to update filter coefficients $[2,7]$. For simplicity, here we refer to stochastic gradient adaptation rule based on MOE criterion:

$$
\left.\mathbf{x}_{1}^{(n+1)}=\mathbf{x}_{1}^{(n)}-2 \mu \mathbf{r}_{M C}^{(n) H}\left(\mathbf{s}_{1}+\mathbf{x}_{1}^{(n)}\right) \mid \mathbf{r}_{M C}^{(n)}-\left(\mathbf{r}_{M C}^{(n) H} \mathbf{s}_{1}\right) \mathbf{s}_{1}\right\rfloor
$$

where

$$
\begin{aligned}
& r_{M C}{ }^{(n)}(t)=\sum_{k=1}^{K} \sum_{l=1}^{L} c_{l}^{(n)} \hat{c}_{l}^{(n)^{*}} A_{k} b_{k}^{(n)} . \\
& \sum_{l^{\prime}=1}^{L} s_{k}\left(t-n T-\tau_{l}+\tau_{l^{\prime}}\right)+n(t)
\end{aligned}
$$

indicates the output of the multipath combiner for the wideband signal.

In this case, $w_{1}$ is the vector that simultaneously passes the desired signature sequence and its replicas with unitary response and is orthogonal to the space spanned by all other codes. So, all multipath components of the signal of interest are combined and MAI is minimized under the constraint $\mathbf{s}_{1}^{T} \mathbf{x}_{1}^{(n)}=0$.

PreBA-SUD consists of a bank of filters matched to each replica of desired signal and, in parallel, an adaptive filter aiming to mitigate MAI associated to that path regardless of other replicas. In such a way, it is suboptimal respect to Post-combining scheme. Updating rule of the $l$-th filter is:

$\mathbf{x}_{1, l}^{(n+1)}=\mathbf{x}_{1, l}^{(n)}-2 \mu \mathbf{r}^{(n) H}\left(\mathbf{s}_{1, l}^{(n)}+\mathbf{x}_{1, l}^{(n)}\right)$.

$\left[\mathbf{r}^{(n)}-\left(\mathbf{r}^{(n) H} \mathbf{S}_{1, l}{ }^{(n)}\right) \mathbf{s}_{1, l}{ }^{(n)}\right]$

where $s_{1, t}{ }^{(n)}(t)=s_{1}\left(t-n T-\hat{\tau}_{l}\right)$ is the locally generated signature waveform properly delayed to match the $l$-th received path.

Then each output of LMMSE filters is multiplied by the relative estimated channel coefficient and summed up to yield the final decision variable (Fig. 1).

$\mathbf{y}_{\text {rre }}^{(n)}=\sum_{l=1}^{L} \mathbf{y}_{l}^{(n)}=\sum_{l=1}^{L} \hat{c}_{l}^{*}\left(\mathbf{w}_{1, I}^{(n)}{ }^{H} \cdot \mathbf{r}^{(n)}\right)$

where $\quad \mathbf{w}_{1, l}^{(n)}=\mathbf{s}_{1, l}^{(n)}+\mathbf{x}_{1, l}^{(n)} \quad$ with the constraint $\mathbf{s}_{1 . l}^{(n) T} \mathbf{x}_{1 . l}^{(n)}=0$. In such a case, $\boldsymbol{w}_{1, l}$ is the vector that passes only the $l$-th path with unity response and is orthogonal to all other codes.

In both schemes, the algorithm step $\mu$, which assures convergence and stability of normalized LMS algorithm, has to be chosen as [2]:

$$
0<\mu<\frac{1}{\mathbf{r}^{(n)} \mathbf{r}^{(n)}}
$$

\section{NUMERICAL RESULTS}

Downlink transmission in multipath fading channel has been considered with:

- BPSK modulation; 
- Number of users: 10;

- Near-far: 0 and $10 \mathrm{~dB}$;

- Gold spreading sequences of length 31;

- Number of equally power paths $(L): 1$ and 2;

- Delay between two adjacent received paths: 1.5 chips;

- Rayleigh classical Doppler spectrum is assumed with Doppler spread $B_{a}=100 \mathrm{~Hz}$. (that implies a fast fading scenario).

Phase estimation error $\Delta \phi_{k . l}^{(n)}=\Delta \phi_{l}^{(n)}$ for each received path has been selected by Uniform distribution in the range $(0, P h)$ where $P h=\{0 \mathrm{deg}, 7.5 \mathrm{deg}, 15 \mathrm{deg}, 30 \mathrm{deg}$, $60 \mathrm{deg}\}$. In simulations the phase error is randomly selected for each symbol interval.

PostBA-SUD, PreBA-SUD and CRD show almost equivalent sensitivity to channel phase estimation errors (Fig. 3) in the case of equal power users as well as in the case of near-far effect. Thus, in the presence of phase errors the proposed receivers maintain better performance than CRD due to their intrinsically nearfar resistance and MAI mitigation capabilities.

Path delay error $\Delta \tau_{k, l^{\prime}}=\Delta \tau_{l^{\prime}}$ is selected also by Uniform distribution in the range $(-\bar{\tau}, \bar{\tau})$ where $\bar{\tau}=\left\{0 T_{c}, 0.1 T_{c}, 0.2 T_{c}, 0.4 T_{c}\right\}$. Error $\Delta \tau_{r^{\prime}}$ is randomly selected for each path and kept constant for the whole simulation.

While PostBA-SUD has always better performance compared to PreBA-SUD if synchronization is perfect, it seems to be more sensitive to timing error than PreBA-SUD and conventional RAKE receiver. Even for a synchronization error of $20 \%$ of the chip period, the degradation is significant (Fig. 4). Larger error implies the loss of diversity gain (Fig 5) and near-far resistance (Fig. 6). PreBA-SUD shows slightly better resistance to synchronization errors, but it approaches CRD if the error increases.

\section{CONCLUSION}

In this paper we have investigated receiver sensitivity to path delay and channel phase estimation errors. The study has focused on the pre and post-combining blind adaptive single-user LMMSE receivers. Comparisons have been made also with conventional RAKE receiver. It has been observed that the blind adaptive LMMSE receivers' performance is not degraded more than that of conventional RAKE receiver in the presence of channel phase estimation errors. Moreover phase errors do not degrade multiple access interference rejection and near-far resistance capabilities.

Post-combining blind adaptive LMMSE receiver has better performance in case of perfect estimation of desired user timing, but turned out to be more sensitive to synchronization errors than Pre-combining scheme and conventional RAKE, in particular in the case of timing error higher than $20 \%$ of one chip period. However, when synchronization error increases both Pre and Post-combining blind adaptive LMMSE receivers approach conventional receiver.

It is therefore important to have good path delay estimator in practical system in order not to loose the benefits of blind adaptive LMMSE detection.

\section{REFERENCES}

[1] M. Honig, U. Madhow, S. Verdù, "Blind Adaptive Multiuser Detection", IEEE Trans. Info. Theory, Vol. 41, No. 4, pp. 944-960, July 1995.

[2] S. Haykin, "Adaptive Filter Theory", 3rd edition, Prentice Hall, Upper Saddle River, NJ, 1996.

[3] S. Chennakeshu, G. J. Saulnier, "Differential Detection of $\pi / 4$-Shifted-DQPSK for Digital Cellular Radio", IEEE Trans. on Vehic. Tech., Vol. 42, n.1, pp. 46-57, Feb. 1993.

[4] U. Madhow, M. Honig, "MMSE Detection of CDMA Signals: Analysis for Random Signature Sequences", Proc. IEEE Int. Symp. on Inf. Theory, San Antonio, TX, p. 49, Jan. 1993.

[5] S. Verdù, "Multiuser Detection", Cambridge UK, Cambridge Univ. Press, 1998.

[6] U. Madhow, M. L. Honig, "MMSE Interference Suppression for Direct-Sequence Spread-Spectrum CDMA", IEEE Trans. Comm., Vol. 42, pp. 3178-3188, Dec. 1994.

[7] M. K. Tsatsanis, Z. D. Xu, "Performance Analysis of Minimum Variance CDMA Receivers", IEEE Trans. on Comm., Vol. 46, n. 11, Nov. 1998, pp. 3014-3022.

[8] M. Juntti, S. Glisic, "Advanced CDMA for wireless communications", in Wireless Communications: TDMA versus CDMA, S. G. Glisic, P. A. Leppänen, Eds. Norwell, MA: Kluver, 1997, ch. 4, pp. 447-490.

[9] M. Latva-aho, M. Juntti, "LMMSE Detection for DS-CDMA Systems in Fading Channels", IEEE Trans. on Comm., Vol. 48, n. 2, Feb. 2000, pp. 194-199. 


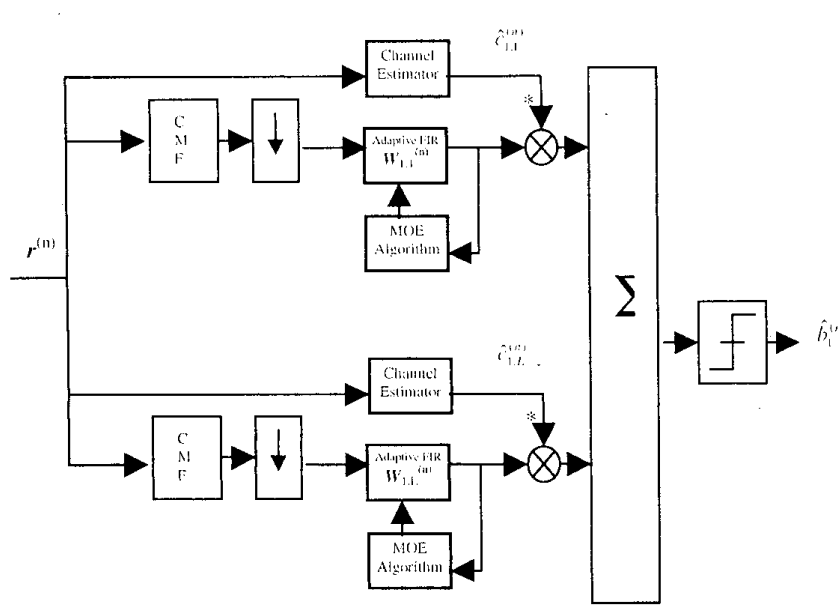

Fig. 1 Pre-combining blind adaptive LMMSE receiver.
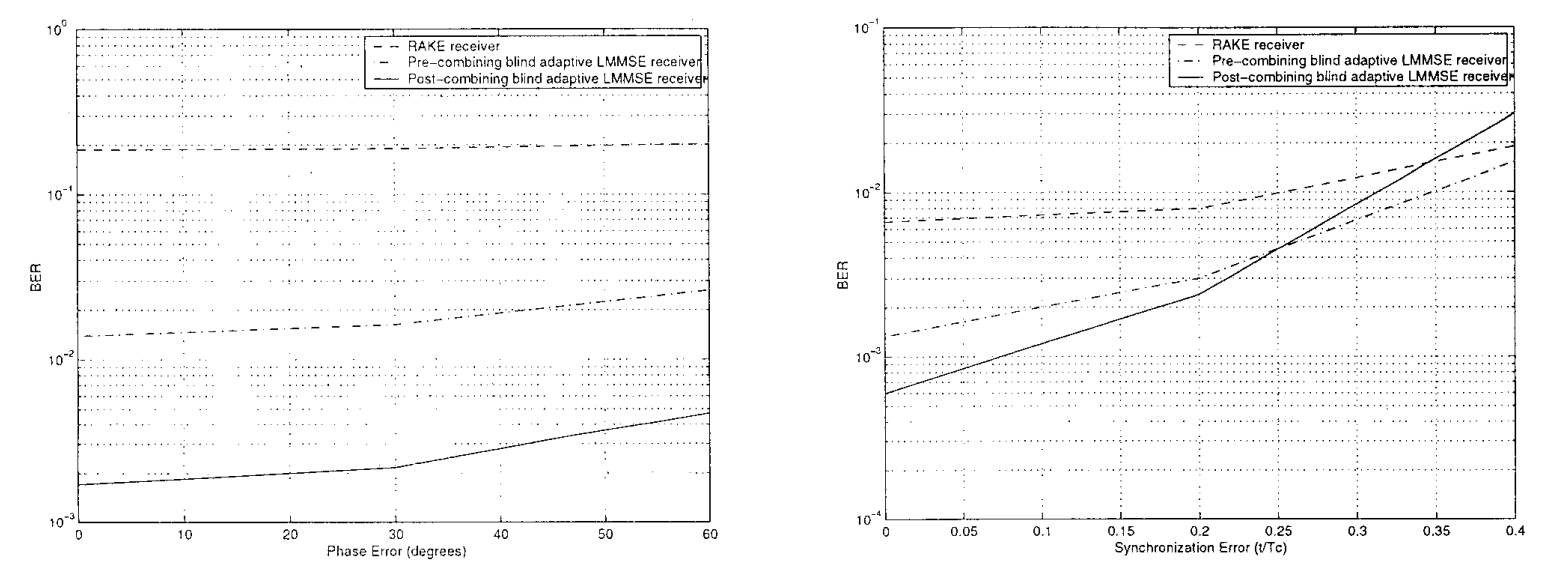

Fig. 3 BER in two-paths fading channel, to users, near-far: $10 \mathrm{~dB}$, perfect synchronization.

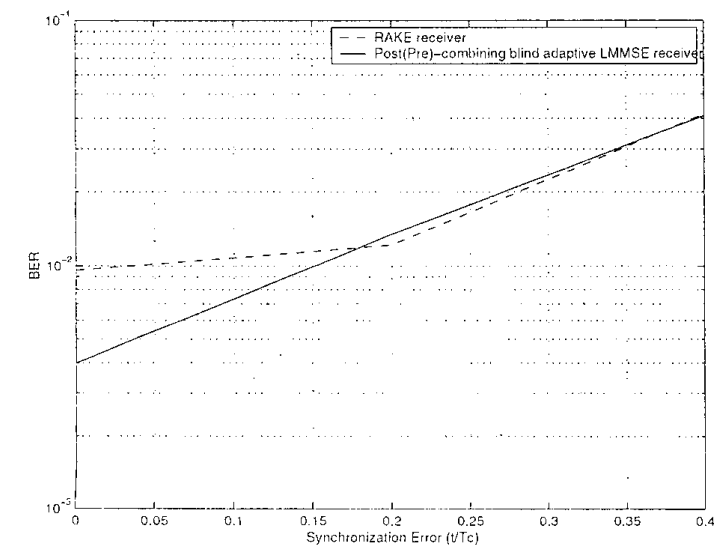

Fig. 4 BER in single-paths fading channel, 10 users, perfect phase estimation. ()-7803-6728-6/(0)/\$10.00 O200I IEEE

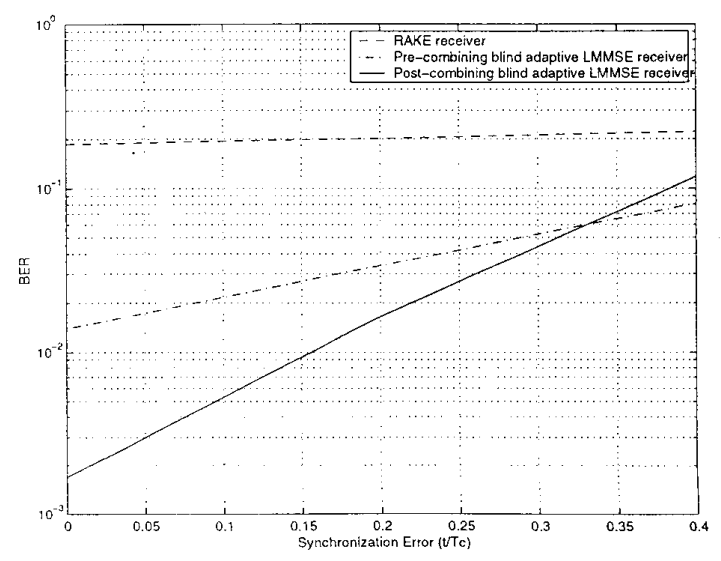

Fig. 6 BER in two-paths fading channel, 10 users, near-far: $10 \mathrm{~dB}$, perfect phase estimation. 